\title{
miR-4782-3p Inhibited Non-Small Cell Lung Cancer growth via USP14
}

\author{
Ning Wua, Chaoxiong Zhang ${ }^{\mathrm{b}, \mathrm{c}}$ Chong Bai ${ }^{\mathrm{a}}$ Yi Pang Han ${ }^{\mathrm{a}}$ Qiang Li ${ }^{\mathrm{a}}$ \\ aDepartment of Respiratory Medicine, Changhai Hospital, Second Military Medical University, \\ Shanghai, ${ }^{\mathrm{b}}$ Department of Centre for Disease Prevention and Control, Chengdu Military Region \\ Chengdu, China; 'These authors contributed equally to this work
}

\author{
Key Words \\ miR-4782-3p • NSCLC ・ USP14 • ZEB2 ・ XIAP
}

\begin{abstract}
Background: Lung cancer is the leading cause of cancer-related mortality worldwide, with near 1.4 million deaths each year. NSCLC accounts for nearly $85 \%$ of all case of lung cancer. MiRNAs play important roles in regulation of gene expression at the post-transcriptional level. MiRNAs profiles may predict prognosis and disease recurrence in early-stage NSCLC. Our previous study proved that over-expression of ubiquitin specific peptidase 14 (USP14), a deubiquitinating enzyme, was associated with favorable prognosis in NSCLC patients and promoted tumor cells proliferation. Here, we tried to identify which miRNAs targeted USP14, and the roles of these miRNAs in NSCLC. Methods: MiR-4782-3p and its potential targeted genes were identified by bioinformatics algorithm. Dual luciferase reporter assay system was used to analyze the interaction between miR-4782-3p and targeted genes. Cell proliferation was assayed by MTT and BdU assay. MiRNAs and mRNA expression were assayed by qRTPCR. USP14 protein level was assayed by Western blot. The role of miR-4782-3p in patients survival was revealed by Kaplan-Meier plot of overall survival. Results: Up-expression of miR4782-3p in NSCLC cells decreased the USP14 expression. Down-expression of miR-4782-3p increased USP14 expression. In NSCLC specimen, Negative correlation between USP14 mRNA level and miR-4782-3p level was identified. Higher miR-4782-3p expression is associated with longer survival. USP14, ZEB2, XIAP overexpression reversed the inhibitory effect of miR-4782$3 p$. Conclusions: High expression of miR-4782-3p was associated with favorable prognosis in NSCLC patients. MiR-4782-3p inhibited cell proliferation in NSCLC by targeting USP14, ZEB2 and $X I A P$.
\end{abstract}

Copyright @ 2014 S. Karger AG, Basel 
Wu et al.: The Role of miR-4782-3p in NSCLC

\section{Introduction}

Approximately 1.6 million new cases of lung cancer are diagnosed each year throughout the world, the mortality related to lung cancer continues to rise [1]. It is the leading cause of cancer-related mortality worldwide, with near 1.4 million deaths each year. Lung cancer has an extremely poor prognosis, with an overall 5 year survival of $16 \%$ in the USA and less than $10 \%$ in the UK [2]. In China, mortality of lung cancer has increased by $465 \%$ during the past 30 years, and such cancer has become the main cause of death in urbanites and the second leading cause of death in rural population after liver cancer [3]. Non-small cell lung cancer (NSCLC) is any type of epithelial lung cancer other than small cell lung carcinoma (SCLC). NSCLC includes adenocarcinoma, large cell carcinoma, bronchioloalveolar carcinoma, and squamous cell carcinoma, and accounts for nearly $85 \%$ of all case of lung cancer $[4,5]$.

MicroRNAs (miRNAs) are a class of endogenous non-coding, single-stranded small regulatory RNA molecules, which are approximately 22 nucleotides in length [6]. MiRNAs inhibit translation and cleave mRNA by base-pairing to the 3 ' untranslated region of the target genes [7-9]. MiRNAs play important roles in regulation of gene expression at the posttranscriptional level. It is estimated that miRNAs can regulate at least 20\%-30\% of all human genes [10], and that an average miRNAs have more than 100 targets [11]. Half of all miRNAs genes are found within or near chromosomal fragile sites, common breakpoints, or minimal regions of loss-of-heterozygosity or amplification [12]. It is known that miRNAs are grossly in NSCLC, and may serve as oncogenes or tumor suppressors [13]. Importantly, recent data showed that specific miRNAs profiles may predict prognosis and disease recurrence in earlystage NSCLC [14-18].

Here, we studied the role of miR-4782-3p in NSCLC, and found miR-4782-3p inhibited cell proliferation in NSCLC. Our study may provide a new therapy target of NSCLC.

\section{Materials and Methods}

\section{Patients}

Surgical specimens from 22 NSCLC patients and matched normal control adjacent lung tissues were obtained postoperatively in 2008 from the Department of Respiratory Medicine, Changhai Hospital, Second Military Medical University (Shanghai, China). All patients gave signed, informed consent for their tissues to be used for scientific research. Ethical approval for the study was obtained from Changhai Hospital, Second Military Medical University (Shanghai, China). All diagnoses were based on pathological and/or cytological evidence. The histological features of the specimens were evaluated by senior pathologists according to the World Health Organization classification criteria. Tissues were obtained before chemotherapy and radiotherapy and were immediately frozen and stored at $-80{ }^{\circ} \mathrm{C}$ prior to qRT-PCR assay. 20 patients had been followed-up for 3 years and complete clinical data were electronically recorded.

\section{Cell culture}

HEK293, human normal lung fibroblast cell line MRC-5, and human NSCLC cell lines (A549, H1299, SPC-A-1, LTEP-A-2 and SK-MES-1) were obtained from the Cell Bank of Chinese Academy of Science (Shanghai, China) and cultured in DMEM medium (Hyclone, South Logan, UT, USA) supplemented with $10 \%$ fetal bovine serum (Hyclone), $2 \mathrm{mM}$ L-glutamine and $100 \mu \mathrm{g} / \mathrm{mL}$ penicillin/streptomycin (Bio Light, Shanghai, China) as described in our previous studies [19].

\section{EdU cell proliferation assay and MTT assay}

The cell proliferation assay were assayed by EdU Flow Cytometry Assay Kits (invitrogen). EdU (5-ethynyl-2'-deoxyuridine) is a nucleoside analog to thymidine and is incorporated into DNA during active DNA synthesis. Detection is based on a click reaction [20], a copper catalyzed covalent reaction between an azide and an alkyne. Flow Cytometry Assay was performed by using CellQuest software (Becton Dickinson, Franklin Lakes, NJ, USA) as described previously [21, 22]. For MTT assay, 500 cells per well were seeded in triplicate in a 96-well plate with complete growth medium. Cells were counted over 5 days using the 
MTT assay (Promega, Fitchburg, WI, USA) as described previously [22, 23]. The data were measured by Microtiter plate reader 570-nm filters (Promega, USA ).

RNA extraction and Real time $q-P C R$

RNA was extracted with Trizol reagent (Invitrogen, Carlsbad, CA, USA) according to the manufacturer's protocol. The cDNA synthesis and real-time qPCR were subsequently performed

using the Qiagen system as described detail in our previous studies $[19,24]$. Real-time quantitative PCR analysis was performed using standard protocols on an Applied Biosystem's 7500 HT sequence Detection System. MiR-4782-3p expression was assessed using a mirVana ${ }^{\mathrm{TM}}$ qRT-PCR miRNA Detection Kit (Ambion, USA). The primer design is based on standard protocols [25], and synthesized by Shengong Company (Shanghai).

Relative mRNA levels of USP14 were normalized to levels of the housekeeping gene GAPDH and calculated by the $2^{-\Delta \Delta c t}$ method. The primers used are as follows: GAPDH (5'-CCATGTTCGTCATGGGTGTGAACCA-3' and 5'-GCCAGTAGAGGCAGGGATGATGTTG-3') and USP14 (5'-GAGT-TGGACCTTT-CCAGA-3' and 5'-TGCTTGCACAG-ATGTGA-3').

MiRNAs mimics, miRNAs antisense oligonucleotides and overexpression plasmids

MiRNAs mimics and miRNAs antisense oligonucleotides (ASO) were obtained from GenePharma (GenePharma, China). MiRNAs mimics, negative control (NC) were transfected into cells at a concentration of 50nM using Lipofectamine 2000 (Invitrogen, Canada) transfection reagent according to the manufacturer's instructions. $48 \mathrm{~h}$ or $72 \mathrm{~h}$ later cells were collected for further experiments. The overexpression plasmid (pcDNA3.1-USP14, pcDNA3.1-ZEB2, pcDNA3.1-XIAP ) were constructed and confirmed by Shengong Company (Shanghai).

\section{USP14 3'UTR reporter analysis}

The USP14 3'UTR reporter plasmid (pRL-USP14) was constructed by Shengong Company (Shanghai). Mutation in the miR-4782-3p seed regions of the USP14 3'UTR were generated using QuikChang Multi site -directed mutagenesis kit (Stratagene). RL reporter plasmids (3.6fmol) and pGL3-control (500ng for normalization; Promega) were transfected with Lipofectamine 2000 (Invitrogen) into HEK293 cells $\left(6 \times 10^{4}\right.$ cells per well). Cells were collected after $48 \mathrm{~h}$ for assay using the Dual Luciferase reporter assay system (Promega) [26].

\section{Western blot and antibodies}

Cells or tumor tissues were harvested, lysed, and blotted as described previously [27]. Membranes were blocked with blocking solution (5\% skim milk in TBST) and incubated with primary antibody, followed by the incubation with appropriate HRP-conjugated secondary antibody. The USP14 antibody (anti-USP14) was purchased from Santa Cruz Biotechnology, Inc. The densitometry of Western blot results was measured using ImageJ software.

\section{MicroRNAs targets prediction}

To identify which miRNAs binding the 3'UTR of USP14 or Targets of miRNAs, TargetScanHuman (http://www.targetscan.org/vert_61/) [10,28-30] is applied.

\section{Apoptosis assay}

Cells were labeled with Annexin V-FITC and propidium iodide (PI) using an apoptosis detecting kit (Invitrogen, Canada) as described previously [31]. Samples were determined by FACS assays and the results were analyzed using CellQuest software (Becton Dickinson, San Jose, CA) [32].

\section{Statistical Analysis}

Data were presented as the mean \pm s.e.m. from at least three independent experiments. The difference between groups were analyzed using two-tailed Student's $t$ test when only two groups were compared. The difference between groups were analyzed using ANOVA when three or more than three groups were compared. Correlation analysis was performed by two-tailed Person's correlation coefficient analysis. Patients survival was determined by Kaplan-Meier analysis. Statistical analyses were performed using SPSS software (version 17.0). $\mathrm{P}<0.05$ was considered significantly different. 


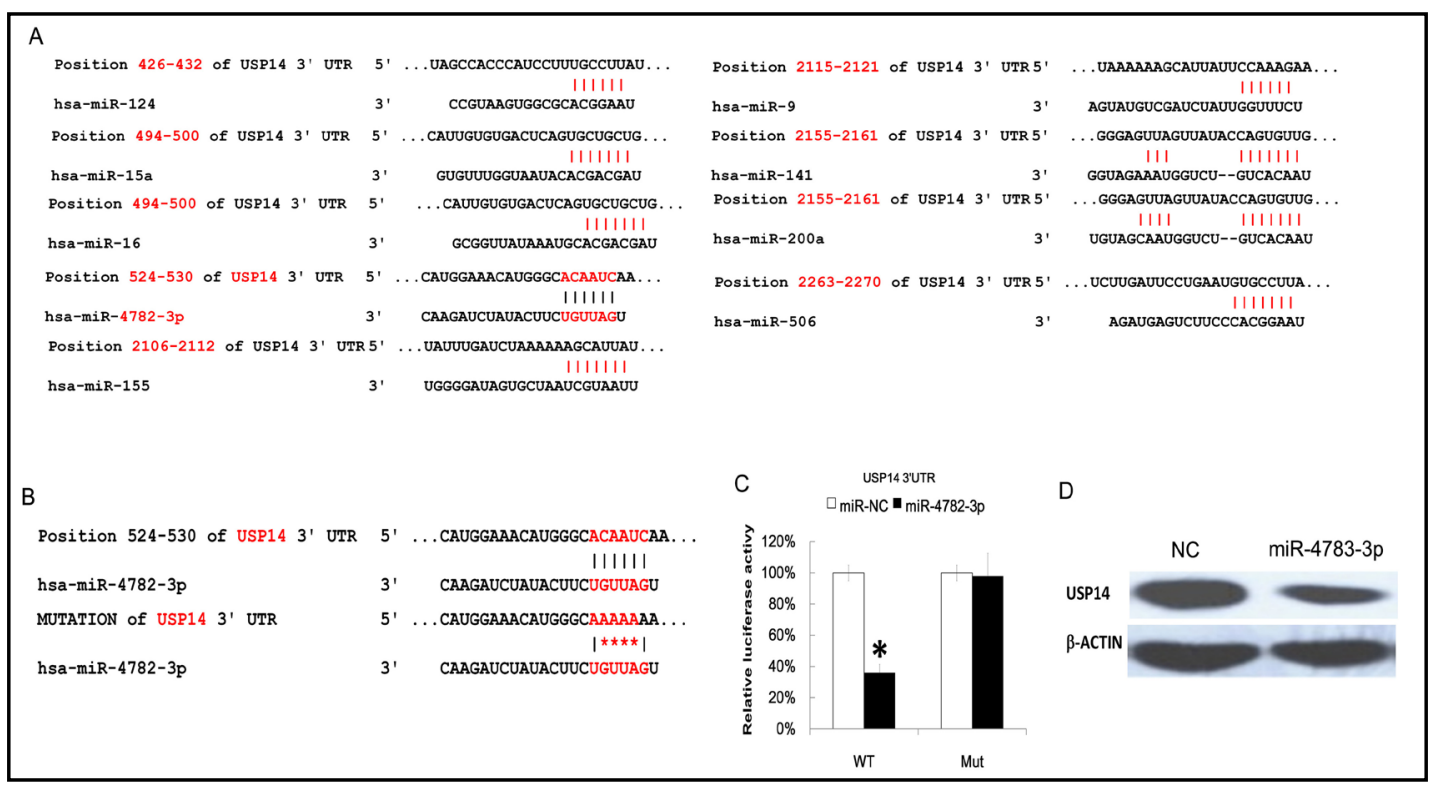

Fig. 1. USP14 expression were down-regulated in mRNA and protein level by miR-4782-3p transfection. 9 putative miRNAs were predicated by bioinformatics analysis (A). The mutation of USP14 3' UTR (miR-4782$3 p$ binding site )(B). The RL reporter plasmids (RL-control, RL-USP14) and miR-4782-3p were co-transfected into HEK293 cells, along with a firefly luciferase reporter (pGL control) for normalization. Luciferase activities were measured after $48 \mathrm{~h}$ transfection. The data are mean \pm s.e.m. of separate transfection $(n=6)$, and shown as the ratio of RL activity of firefly luciferase activity. The luciferase activity in negative control group was arbitrarily defined as $100 \%$ (C). $48 \mathrm{~h}$ after the miR-4782-3p transfection, the USP14 protein in HEK293 cells were assayed by Western blot (D). ${ }^{*} \mathrm{P}<0.05$.

\section{Results}

miR-4782-3p inhibited USP14 expression

Our previous study proved that over-expression of USP14 was associated with poor prognosis in NSCLC patients and promoted tumor cell proliferation [19]. To identified which miRNAs binding the 3'UTR of USP14, bioinformatics algorithm is applied. We found that 9 potential miRNAs may probably bind the 3'UTR of USP14 (Fig. 1A). Of these 9 miRNAs, the role of miR-4782-3p has not been investigated. To confirm whether miR-4782-3p could target USP14, the putative miR-4782-3p binding site in 3' UTR USP14 was mutated (Fig. 1B). WT or mutated USP14 3' UTR were cloned into luciferase reporter plasmid. MiR-4782-3p mimics and reporter plasmid were co-transfected into HEK293 cells. We found that miR4782-3p inhibited renilla luciferase (RL) reporter genes, and mutation of miR-4782-3p binding sites in USP14 prevented down-regulation of reporters by miR-4782-3p mimics (Fig. 1C). Next, we transfected HEK293 cells with miR-4782-3p mimics, $48 \mathrm{~h}$ later, we found that the USP14 protein expression were inhibited (Fig. 1D). So, our data indicated that miR-47823 p inhibited USP14 expression.

\section{Correlations between miR-4782-3p, USP14 and overall survival in NSCLC patients}

To confirm the correlations between miR-4782-3p and USP14, the miR-4782-3p expression and USP14 in human normal lung tissue, human normal lung fibroblast cell line (MRC-5), 7 human NSCLC cell lines (A549, H1299, SPC-A-1, LTEP-A-2 and SK-MES-1) were analyzed. We found that miR-4782-3p expression in NSCLC cell lines was lower than in human normal lung tissue and MRC-5 (Fig. 2A), and there was a negative correlation between USP14 mRNA level and miR-4782-3p level (Fig. 2B). 22 pairs of NSCLC tumor tissues and matched normal adjacent tissue analysis revealed that in 17 pairs, the miR-4782-3p in NSCLC tumor tissues expression was lower than matched normal adjacent tissue, and in 3 pairs, miR- 


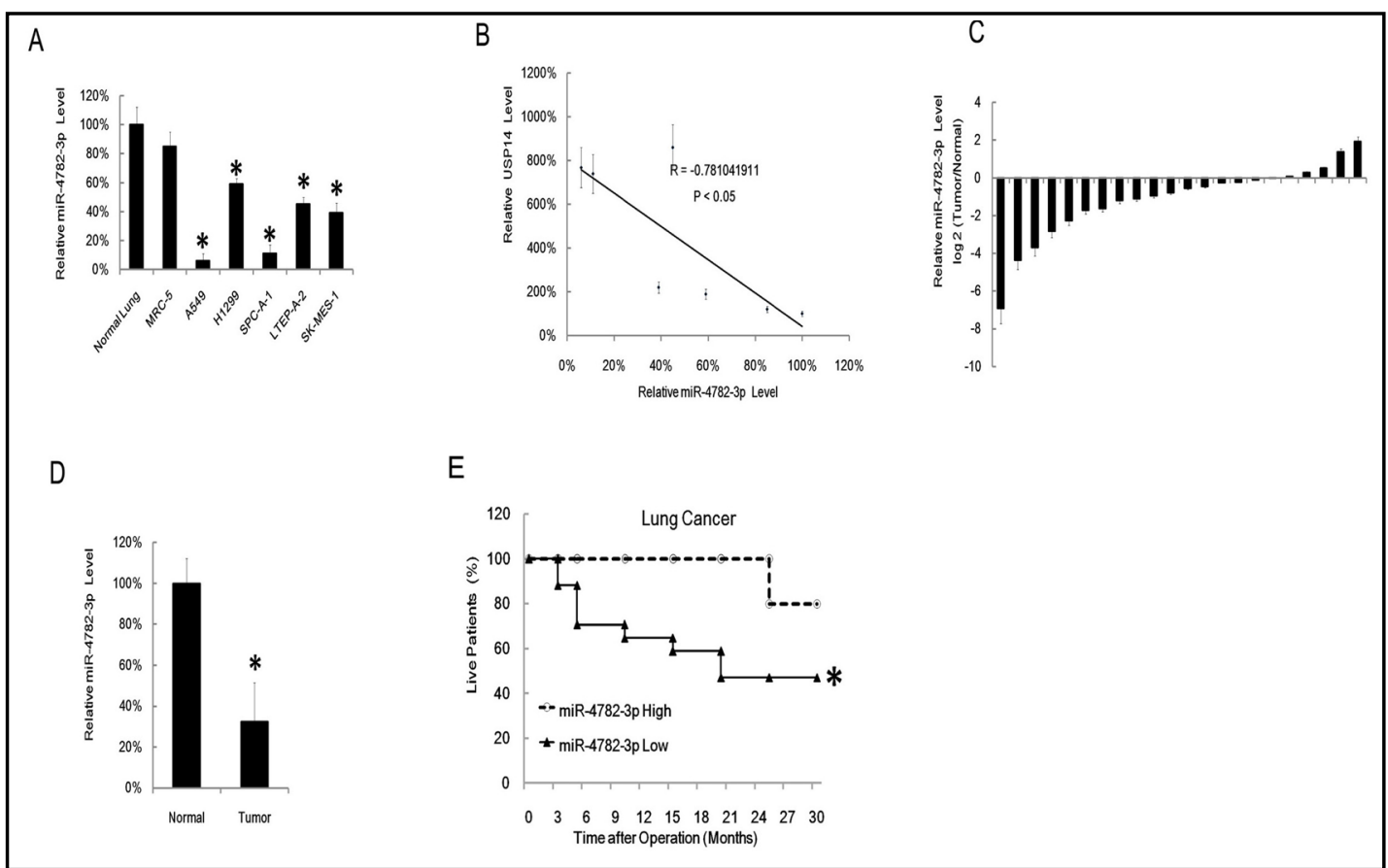

Fig. 2. Correlation between miR-4782-3p, USP14 and overall survival in NSCLC patients. qRT-PCR analysis of miR-4782-3p expression in normal lung and NSCLC cell line. 3 normal lung tissues came from three patient. The miR-4782-3p expression in normal lung were arbitrary defined as $100 \%$. Data are mean \pm s.e.m. of three separate experiments (A). Inverse correlation between miR-4782-3p and USP14 mRNA in the 6 NSCLC cell lines and normal lung tissue (B). qRT-PCR analysis of miR-4782-3p in 22 NSCLC tissues (C). The general expression of miR-4782-3p in 22 NSCLC tumor tissues were compared with matched normal adjacent tissue, the expression in normal tissue were arbitrary defined as $100 \%$. Data are mean \pm s.e.m. of three separate experiments (D). Kaplan-Meier plot of overall survival in NSCLC patient post-operation according the expression of miR-4782-3p (E). ${ }^{*} \mathrm{P}<0.05$.

4782-3p in NSCLC tumor tissues expression was higher than matched normal adjacent tissue (Fig. 2C). Then the general expression of miR-4782-3p in 22 NSCLC tumor tissues was compared with matched normal adjacent tissues, We observed that the mean expression of miR-4782-3p in NSCLC tumor tissues was lower than in normal adjacent tissue (Fig. 2D). To evaluate the clinical significance of miR-4782-3p in NSCLC, we investigated whether the level of miR-4782-3p expression were associated with overall survival in NSCLC patients. 20 NSCLC patients had been followed-up for 3 years. During the 3-year follow-up period, 7 out of $20(35 \%)$ patients died as a result of disease progression. Survival curve showed that patient with high miR-4782-3p level (10 case) had a significantly longer overall survival than those with low miR-4782-3p level (10 case) (Fig. 2E).

The role of miR-4782-3p in cell proliferation and apoptosis

Data above indicated that miR-4782-3p may play an important role in NSCLC. We over-expressed the miR-4782-3p in A549, SPC-A-1 by miRNAs mimics transfection. After 48h transfection, miR-4782-3p in A549 and SPC-A-1 reached the maximum value (Fig. 3A), and the USP14 level were inhibited (Fig. 3B). MTT assay showed that up-regulation of miR4782-3p in A549, SPC-A-1 inhibited cells proliferation (Fig. 3C). EdU assay also showed that miR-4782-3p inhibited A549, SPC-A-1 proliferation. The percent of EdU positive cells in miR-4782-3p pretreated group was lower than in miR-NC pretreated group (Fig. 3D,E). MiR4782-3p showed more inhibitory effect on cells proliferation in A549 than in SPC-A-1(Fig. $3 \mathrm{E})$. Cell apoptosis assay revealed that miR-4782-3p induced more cell apoptosis in A549 and SPC-A-1than miR-NC (Fig. 3F). 




Fig. 3. miR-4782-3p inhibited cell proliferation and induced cell apoptosis. A549 and SPC-A-1 cells $\left(6 \times 10^{5}\right.$ cells/well) were transfected with miR-4782-3p mimics or miR-NC (NC) separately. 48h or 72h later, the miR-4782-3p expression were assayed by qRT-PCR. Data were normalized to U6 small nuclear RNA. The miR-4782-3p expression in normal lung were arbitrary defined as $100 \%$. Data are mean \pm s.e.m. of three separate experiments (A). 48h after miR-4782-3p transfection, the USP14 expression level were assayed by qRT-PCR. Data were normalized to GAPDH mRNA. The USP14 expression in control group were arbitrary defined as $100 \%$ (B). After the miR-4782-3p transfection, the cell proliferation were assayed by MTT at the indicted time point. Data are mean \pm s.e.m. of three separate experiments (C). After $48 \mathrm{~h}$ transfection, A549 and SPC-A-1 cells were treated with $10 \mu \mathrm{M}$ of EdU for 1 hour, then were assayed by flow cytometry. The percent of EdU positive A549 cells in miR-NC pretreated group is 28.6\%, and the percent of EdU positive A549 cells in miR-4782-3p pretreated group is $15.2 \%$ (D, E). The percent of EdU positive SPC-A-1 cells in miR-NC pretreated group is $24.3 \%$, and the percent of EdU positive SPC-A-1 cells in miR-4782-3p pretreated group is $17.1 \%$. Data are mean \pm s.e.m. of three separate experiments(E). The cell apoptosis rate after 48 h miR-4782-3p transfection in A549 and SPC-A-1 cells. The apoptosis rate in miR-NC group was arbitrarily defined as $100 \%$. Data are mean \pm s.e.m. of three separate experiments $(\mathrm{F})$. ${ }^{*} \mathrm{P}<0.05$.

\section{Down-regulation of miR-4782-3p promoted $H 1299$ and MRC-5 proliferation}

Data above showed that H1299 and MRC-5 have a high miR-4782-3p level (Fig. 1A), thus we down-regulated the miR-4782-3p level by miR-4782-3p ASO transfection (Fig. 4A). $48 \mathrm{~h}$ later, qRT-PCR were performed to assay the USP14 mRNA level, and MTT assay was performed to assay cells proliferation. We found that miR-4782-3p ASO increased the USP14 mRNA expression(Fig. 4B) and promoted cell proliferation in H1299 and MRC-5 (Fig. 4C). Then, the role of miR-4782-3p ASO in H1299 and MRC- 5 were confirmed by EdU test, we found that the miR-4782-3p ASO increased the percent of EdU positive cells in H1299 and MRC-5 (Fig. 4D). 




Fig. 4. miR-4782-3p ASO transfection promoted H1299 and MRC-5 proliferation. H1299 and MRC-5 cells $\left(6 \times 10^{5}\right.$ cells/well) were transfected with miR-4782-3p ASO or miR-NC ASO separately. $48 \mathrm{~h}$ later, the miR4782-3p expression was assayed by qRT-PCR. Data were normalized to U6 small nuclear RNA. The miR4782-3p expression in normal lung was arbitrary defined as $100 \%$. Data are mean \pm s.e.m. of three separate experiments (A). 48h later, the USP14 mRNA expression was assayed by qRT-PCR. Data were normalized to GAPDH mRNA. The USP14 expression in control group were arbitrary defined as $100 \%$ (B). After the miR4782-3p ASO transfection, Cell proliferation was assayed by MTT at the indicted time point. Data are mean \pm s.e.m. of three separate experiments (C). After 48h transfection, H1299 and MRC-5 cells were treated with $10 \mu \mathrm{M}$ of EdU for 1 hour, then were assayed by flow cytometry. The percent of EdU positive cells were compared. Data are mean \pm s.e.m. of three separate experiments (D). ${ }^{*} \mathrm{P}<0.05$.

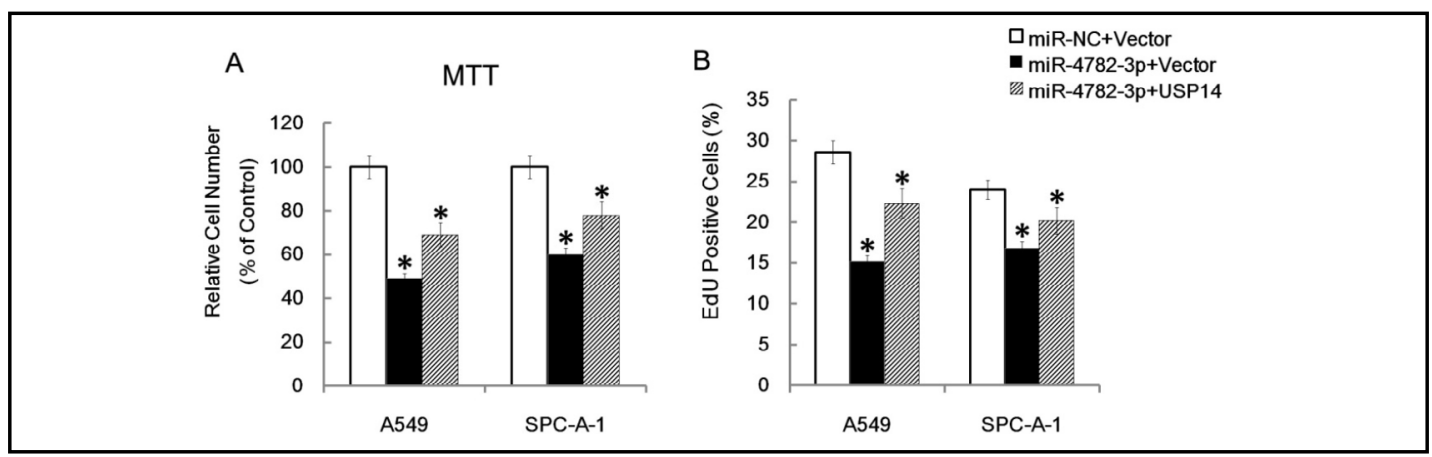

Fig. 5. USP14 overexpression partly reduced the inhibitory effect of miR-4782-3p. A549 and SPC-A-1 cells $\left(6 \times 10^{5}\right.$ cells/well) were transfected with miR-4782-3p or miR-NC (NC) separately. $12 \mathrm{~h}$ later, these cell were transfected with empty plasmid, or USP14 overexpression plasmid (pcDNA3.1-USP14). Then the MTT and EdU proliferation assay were performed. The OD value from MTT assay were presented as the relative cell number. The relative cell number in miR-NC+Vector (negative control) was defined as 100\% (A). EdU proliferation assay showed the similar results (B).Data are mean \pm s.e.m. of three separate experiments. ${ }^{*} \mathrm{P}<0.05$.

UPS14 played a part role in the inhibitory effect of miR-4782-3p

Our previous data proved that over-expression of USP14 promoted NSCLC proliferation [19]. Data above indicated that miR-4782-3p inhibited USP14 expression (Fig 1C,D), and 




Fig. 6. miR-4782-3p inhibited another genes. 10 putative target genes were predicated by bioinformatics analysis (A). The RL reporter plasmids (RL-control, RL-GAPDH, RL-USP14, RL-ZEB2, RL-XIAP, RL-RAB35, RL-LEF1, RL-ELK1, RL-S0X14, RL-KLF13, RL-KLF16, RL-STAB1, RL-FBXW7) and miR-4782-3p or miR-NC were co-transfected into HEK293 cells, along with a firefly luciferase reporter (pGL control) for normalization. Luciferase activities were measured after $48 \mathrm{~h}$. Then the ratio of RL activity of firefly luciferase activity in miR-4782-3p treated group were calculated and compared with the ratio in miR-NC group (which was arbitrary defined as $100 \%$ ). Data are mean \pm s.e.m. of three separate experiments. ${ }^{*} \mathrm{P}<0.05$.

inhibited cell proliferation and induced cell apoptosis (Fig. 3B,C,D). So, we guessed that miR4782-3p exerted its role in NSCLC by interacting with USP14. To confirm the hypothesis, we over-expressed the USP14 after the miR-4782-3p transfection, we found that USP overexpression could partly reduced the inhibitory effect of miR-4782-3p (Fig.5 A,B). So, we guessed that another target genes of miR-4782-3p existed.

\section{Another target genes of miR-4782-3p}

To identified the potential target genes of miR-4782-3p, bioinformatics algorithm methods were used, and hundreds of genes were predicted (Table S1). Based on our interest, we chose 10 putative genes (ZEB2, XIAP, RAB35, LEF1, ELK1, SOX14, KLF13, KLF16, STAB1, FBXW7) for further study (Fig. 6A). Then 3'UTR of these genes were cloned into luciferase reporter plasmid. We co-transfected miR-4782-3p with these reporter genes into HEK293 cells, and found that there are five gene which is significantly inhibited by miR-4782-3p besides USP14 (Fig. 6B).

USP14, ZEB2, XIAP overexpression reversed the inhibitory effect of miR-4782-3p

As USP14, ZEB2, XIAP showed the most effect in reporter gene assay, we chose the three genes for further analysis. Data above showed miR-4782-3p inhibited the NSCLC proliferation (Fig. 3B.C). We found co-transfection of USP14, ZEB2, XIAP overexpression plasmid reduced the inhibitory effect of miR-4782-3p, and more importantly, co-transfection of the three USP14, ZEB2, XIAP overexpression plasmid together totally reversed the inhibitory effect of miR-4782-3p (Fig. 7A). Unsurprisingly, EdU assay also showed that overexpression of USP14, ZEB2, XIAP could restore the cell proliferation to the same level of negative control in SPC-A-1 cells, and MTT assay also showed the similar result in both cell lines (Fig. 7B).

\section{Discussion}

To the best of our knowledge, this was perhaps the first report demonstrating the role of miR-4782-3p in NSCLC. Previous data showed that a four-microRNA signature (hsa-let7a, hsa-miR-221, hsa-miR-372, and hsa-miR-182) that is associated with survival and cancer 
Fig. 7. USP14, ZEB2, XIAP overexpression plasmid together totally reversed the inhibitory effect of miR4782-3p. A549 cells $\left(6 \times 10^{5}\right.$ cells/well) were co-transfected with miR-4782-3p and overexpression plasmid (pcDNA3.1-USP14, pcDNA3.1-ZEB2, pcDNA3.1-XIAP) together or separately. After $48 \mathrm{~h}$ transfection, cells were treated with $10 \mu \mathrm{M}$ of EdU for 1 hour as protocol indicated. Then, the pretreated cells were assayed by flow cytometry. The percent of positive cells were calculated. Data are mean \pm s.e.m. of three separate experiments (A). A549 and SPC-A-1 cells $\left(6 \times 10^{5}\right.$ cells/well $)$ were co-transfected with miR4782-3p and overexpression plasmid (pcDNA3.1-USP14, pcDNA3.1-ZEB2, pcDNA3.1-XIAP) together. After $48 \mathrm{~h}$ transfection, cells were
A

A549
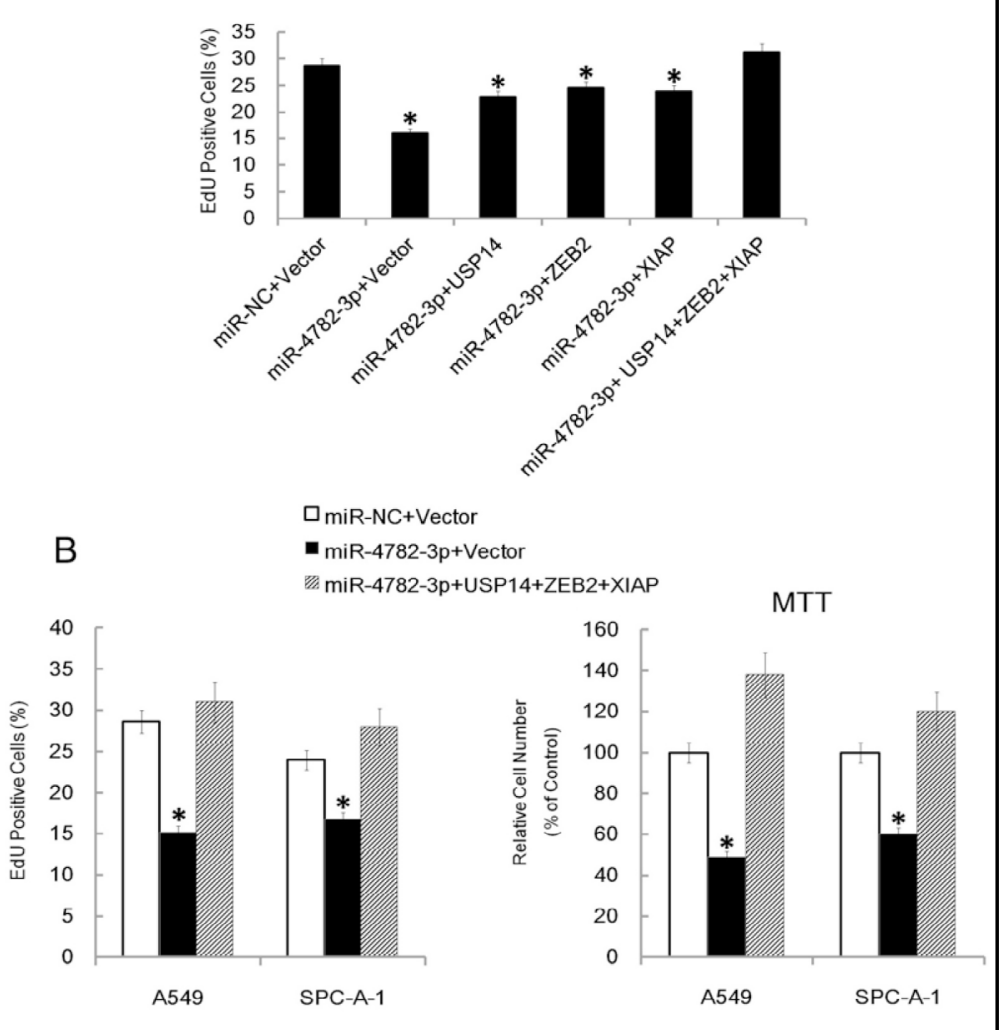

treated with $10 \mu \mathrm{M}$ of EdU for 1 hour, then, were assayed by flow cytometry. The percent of positive cells were calculated (C). Or these cells were assayed by MTT test. All of these data are mean \pm s.e.m. of three separate experiments. ${ }^{*} \mathrm{P}<0.05$.

relapse in NSCLC patients. More importantly, the five microRNAs signature can predict patient survival within cancer stages and histological subgroups of NSCLC patients [15]. Our data showed that patient with high miR-4782-3p level (10 case) had a significantly longer overall survival than those with low miR-4782-3p level $(10$ case $)(\mathrm{P}<0.05)$. Our finding may added a potential miRNA predictor for clinical practice.

Here, we proved that miR-4782-3p inhibited NSCLC proliferation by interacting with USP14, ZEB2, XIAP. Our previous data proved that over-expression of USP14 promoted tumor cell proliferation [19]. ZEB2 is a key mediator of epithelial to mesenchymal transition (EMT). It is known that the miR-200 family (miR-200a, miR200b, miR-200c, miR-141 and miR-429) and miR-205 regulated EMT by targeting ZEB1 and SIP1(ZEB2), inhibition of microRNAs was sufficient to induce EMT in a process requiring up-regulation of ZEB1 and/ or SIP1(ZEB2) [33]. Our data showed that ZEB2 played a part role in the inhibitory effect of miR-4782-3p on cell proliferation. We guessed that may miR-4782-3p could regulate epithelial to mesenchymal transition via $Z E B 2$, and we wondered whether miR-200 family and miR-4782-3p have collaboration or interaction during epithelial to mesenchymal, or in the regulation of cell proliferation. These questions remained further investigation. The X-linked inhibitor of apoptosis (XIAP) is the only cellular protein that has evolved to potently inhibit the enzymatic activity of mammalian caspases and promotes resistance to apoptosis [34]. Previous reports demonstrated that miR-23a or miR-200bc/429 were associated in altered conditions such as cerebral ischemia [35] or chemotherapy resistance in highly selected cancer cell clones [36], respectively. To the best of our knowledge, this is the first 
Wu et al.: The Role of miR-4782-3p in NSCLC

report demonstrating a novel role for miR-4782-3p in determining proliferation in cancer cells. Whether miR-4782-3p could regulate apoptotic cell death resistance via XIAP needed further investigation.

In conclusion, we proved that miR-4782-3p inhibited cell proliferation in NSCLC by targeting USP14, ZEB2 and XIAP. Our study may provide a potential target for NSCLC therapy.

\section{Conflict of Interest}

The authors have declared that no competing interests exist.

\section{Acknowledgements}

This work was supported by grants from the National Natural Science Foundation of China (Grants No. 81201850).

\section{Reference}

1 Ramalingam SS, Owonikoko TK, Khuri FR: Lung cancer: New biological insights and recent therapeutic advances. CA Cancer J Clin 2011;61:91-112.

$>2$ Lung cancer: A global scourge. Lancet 2013;382:659.

7 Wen C, Dehnel T: China wrestles with lung cancer. Lancet Oncol 2011;12:15.

-4 Wakelee HA, Chang ET, Gomez SL, Keegan TH, Feskanich D, Clarke CA, Holmberg L, Yong LC, Kolonel LN, Gould MK, West DW: Lung cancer incidence in never smokers. J Clin Oncol 2007;25:472-478.

-5 Fujii T, Dracheva T, Player A, Chacko S, Clifford R, Strausberg RL, Buetow K, Azumi N, Travis WD, Jen J: A preliminary transcriptome map of non-small cell lung cancer. Cancer Res 2002;62:3340-3346. Ambros V: Micrornas: Tiny regulators with great potential. Cell 2001;107:823-826.

Kim VN, Han J, Siomi MC: Biogenesis of small rnas in animals. Nat Rev Mol Cell Biol 2009;10:126-139. Bartel DP: Micrornas: Target recognition and regulatory functions. Cell 2009;136:215-233.

Valencia-Sanchez MA, Liu J, Hannon GJ, Parker R: Control of translation and mrna degradation by mirnas and sirnas. Genes Dev 2006;20:515-524.

10 Lewis BP, Burge CB, Bartel DP: Conserved seed pairing, often flanked by adenosines, indicates that thousands of human genes are microrna targets. Cell 2005;120:15-20.

11 Griffiths-Jones S, Saini HK, van Dongen S, Enright AJ: Mirbase: Tools for microrna genomics. Nucleic Acids Res 2008;36:D154-158.

-12 Calin GA, Sevignani C, Dumitru CD, Hyslop T, Noch E, Yendamuri S, Shimizu M, Rattan S, Bullrich F, Negrini M, Croce CM: Human microrna genes are frequently located at fragile sites and genomic regions involved in cancers. Proc Natl Acad Sci U S A 2004;101:2999-3004.

13 Croce CM: Causes and consequences of microrna dysregulation in cancer. Nat Rev Genet 2009;10:704-714.

14 Yanaihara N, Caplen N, Bowman E, Seike M, Kumamoto K, Yi M, Stephens RM, Okamoto A, Yokota J, Tanaka T, Calin GA, Liu CG, Croce CM, Harris CC: Unique microrna molecular profiles in lung cancer diagnosis and prognosis. Cancer Cell 2006;9:189-198.

15 Yu SL, Chen HY, Chang GC, Chen CY, Chen HW, Singh S, Cheng CL, Yu CJ, Lee YC, Chen HS, Su TJ, Chiang CC, Li HN, Hong QS, Su HY, Chen CC, Chen WJ, Liu CC, Chan WK, Li KC, Chen JJ, Yang PC: Microrna signature predicts survival and relapse in lung cancer. Cancer Cell 2008;13:48-57.

16 Raponi M, Dossey L, Jatkoe T, Wu X, Chen G, Fan H, Beer DG: Microrna classifiers for predicting prognosis of squamous cell lung cancer. Cancer Res 2009;69:5776-5783.

17 Seike M, Goto A, Okano T, Bowman ED, Schetter AJ, Horikawa I, Mathe EA, Jen J, Yang P, Sugimura H, Gemma A, Kudoh S, Croce CM, Harris CC: Mir-21 is an egfr-regulated anti-apoptotic factor in lung cancer in neversmokers. Proc Natl Acad Sci U S A 2009;106:12085-12090. 
Wu et al.: The Role of miR-4782-3p in NSCLC

18 Patnaik SK, Kannisto E, Knudsen S, Yendamuri S: Evaluation of microrna expression profiles that may predict recurrence of localized stage i non-small cell lung cancer after surgical resection. Cancer Res 2010;70:36-45.

19 Wu N, Liu C, Bai C, Han YP, Cho WC, Li Q: Over-expression of deubiquitinating enzyme usp14 in lung adenocarcinoma promotes proliferation through the accumulation of beta-catenin. Int J Mol Sci 2013;14:10749-10760.

-20 Breinbauer R, Kohn M: Azide-alkyne coupling: A powerful reaction for bioconjugate chemistry. Chembiochem 2003;4:1147-1149.

21 Liu C, Li B, Cheng Y, Lin J, Hao J, Zhang S, Mitchel RE, Sun D, Ni J, Zhao L, Gao F, Cai J: Mir-21 plays an important role in radiation induced carcinogenesis in balb/c mice by directly targeting the tumor suppressor gene big-h3. Int J Biol Sci 2011;7:347-363.

-22 Liu C, Gao F, Li B, Mitchel RE, Liu X, Lin J, Zhao L, Cai J: Tlr4 knockout protects mice from radiation-induced thymic lymphoma by downregulation of il6 and mir-21. Leukemia 2011;25:1516-1519.

23 Liu C, Zhou C, Gao F, Cai S, Zhang C, Zhao L, Zhao F, Cao F, Lin J, Yang Y, Ni J, Jia J, Wu W, Zhou L, Cui J, Zhang W, Li B, Cai J: Mir-34a in age and tissue related radio-sensitivity and serum mir-34a as a novel indicator of radiation injury. Int J Biol Sci 2011;7:221-233.

24 Wu N, Gu HJ, Li Q: Effects of antidiabetic drug metformin on the migration and invasion abilities of human pulmonary adenocarcinoma a549 cell line in vitro. J Thorac Dis 2010;2:76-80.

-25 Chen C, Ridzon DA, Broomer AJ, Zhou Z, Lee DH, Nguyen JT, Barbisin M, Xu NL, Mahuvakar VR, Andersen MR, Lao KQ Livak KJ, Guegler KJ: Real-time quantification of micrornas by stem-loop rt-pcr. Nucleic Acids Res 2005;33:e179.

-26 Grentzmann G, Ingram JA, Kelly PJ, Gesteland RF, Atkins JF: A dual-luciferase reporter system for studying recoding signals. RNA 1998;4:479-486.

-27 Hou J, Lin L, Zhou W, Wang Z, Ding G, Dong Q, Qin L, Wu X, Zheng Y, Yang Y, Tian W, Zhang Q, Wang C, Zhuang SM, Zheng L, Liang A, Tao W, Cao X: Identification of mirnomes in human liver and hepatocellular carcinoma reveals mir-199a/b-3p as therapeutic target for hepatocellular carcinoma. Cancer Cell 2011;19:232-243.

-28 Friedman RC, Farh KK, Burge CB, Bartel DP: Most mammalian mrnas are conserved targets of micrornas. Genome Res 2009;19:92-105.

29 Grimson A, Farh KK, Johnston WK, Garrett-Engele P, Lim LP, Bartel DP: Microrna targeting specificity in mammals: Determinants beyond seed pairing. Mol Cell 2007;27:91-105.

30 Garcia DM, Baek D, Shin C, Bell GW, Grimson A, Bartel DP: Weak seed-pairing stability and high target-site abundance decrease the proficiency of lsy- 6 and other micrornas. Nat Struct Mol Biol 2011;18:1139-1146.

31 Liu C, Zhang C, Mitchel RE, Cui J, Lin J, Yang Y, Liu X, Cai J: A critical role of toll-like receptor 4 (tlr4) and its' in vivo ligands in basal radio-resistance. Cell Death Dis 2013;4:e649.

-32 Lu J, Wen M, Huang Y, He X, Wang Y, Wu Q, Li Z, Castellanos-Martin A, Abad M, Cruz-Hernandez JJ, Rodriguez CA, Perez-Losada J, Mao JH, Wei G: C2orf40 suppresses breast cancer cell proliferation and invasion through modulating expression of $m$ phase cell cycle genes. Epigenetics 2013;8:571-583.

33 Gregory PA, Bert AG, Paterson EL, Barry SC, Tsykin A, Farshid G, Vadas MA, Khew-Goodall Y, Goodall GJ: The mir-200 family and mir-205 regulate epithelial to mesenchymal transition by targeting zeb1 and sip1. Nat Cell Biol 2008;10:593-601.

34 Kashkar H: X-linked inhibitor of apoptosis: A chemoresistance factor or a hollow promise. Clin Cancer Res 2010;16:4496-4502.

-35 Siegel C, Li J, Liu F, Benashski SE, McCullough LD: Mir-23a regulation of x-linked inhibitor of apoptosis (xiap) contributes to sex differences in the response to cerebral ischemia. Proc Natl Acad Sci USA 2011;108:11662-11667.

-36 Zhu W, Xu H, Zhu D, Zhi H, Wang T, Wang J, Jiang B, Shu Y, Liu P: Mir-200bc/429 cluster modulates multidrug resistance of human cancer cell lines by targeting bcl 2 and xiap. Cancer Chemother Pharmacol 2012;69:723-731. 